\title{
A Dual-band Underwater Image Denoising and Enhancement Algorithm
}

\author{
Guoliang Yang, Fuyuan Peng, Kun Zhao \\ Department of Electronics and Information Engineering, \\ Huazhong University of Science and Technology \\ Wuhan, china \\ garden2eyes@163.com
}

\begin{abstract}
In this paper, we proposed a dual-band underwater image denoising and enhancement algorithm, first the original image was decomposed into high-frequency part $\mathrm{H}$ and lowfrequency part $L$, and then $H$ was filtered into $F$ by mean shift algorithm which was improved by using the intermediate iteration results. A contrast enhancement method was proposed based on the haze imaging model and was applied on $L$ and $F$. experiment results demonstrate the effectiveness of the proposed algorithm.
\end{abstract}

Keywords-Underwater image, Image enhancement, Mean shift

\section{INTRODUCTION}

Because of poor imaging condition, bad influence of light absorption and scattering by water molecule, underwater images usually have lower contrast and stronger noise, this is a major problem for many applications of computer vision in underwater enviroment. Traditional algorithms, such as histgram equlization and homomorphic filtering, were unable to resolve this problem. In this paper, we proposed an image enchancement algorithm for underwater image, the aim of enhancement algorithm is to enhance the detail, sharpen the edge and filter the noise. First the original image was decomposed into highfrequency part and low-frequency part, low-frequency part represents the illumination intensity, high-frequency part represnets the image detail and also the noise. And then, filter the high-frequency part using mean shift method which can maintain the edge while eliminating the noise. the general mean shift filtering method is computationlly expensive, so we improved it by utilizing the intermediate iteration result, which maintain the effectiveness while reduce the iteration counts greatly. howerver after the filtering, the image is still fuzzy and misty due to the light absorption and scattering by water molecule. Illumined by haze removal method, we proposed a contrast enhancement algorithm based on haze imaging model, and the experiments demonstrate the effectiveness of the proposed algorithm.

\section{GAUSSIAN FILTERING FOR UNDERWATER}

\section{IMAGE}

Figure 1(a)(cited from [1]) showed a typical underwater image, the object is a man-made structure, it is very fuzzy and misty with uneven distributed lighting and strong noise, and seriously influnced the subsequent procedure. Naturally, we want to eliminate the noise and remove the mist. Here, we first decomposed the original image into high-frequency part and low-frequency part through Gaussian filter.

$I_{\text {low }}(x)=I(x)^{*} G(x, \sigma), I_{\text {high }}(x)=I(x)-I_{\text {low }}(x)$, where $I$ is the original underwater image, $I_{\text {low }}$ is the low-frequency part and $I_{\text {high }}$ is the high-frequency part, $G(x, \sigma)$ is the Gaussian function, $\sigma$ is the standard variation which is set to 8 in the experiment. Figure 1(b) represents the lowfrequency image and figure 1(c) represents the highfrequency image. It is obvious that $I_{\text {low }}$ remain the lighting distribution, while $I_{\text {high }}$ maintain the detail and also noise.

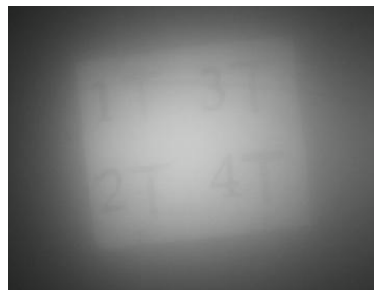

(a) original image

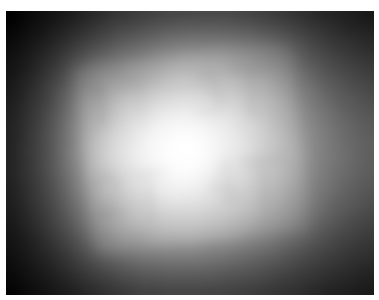

(b) low-frequency part

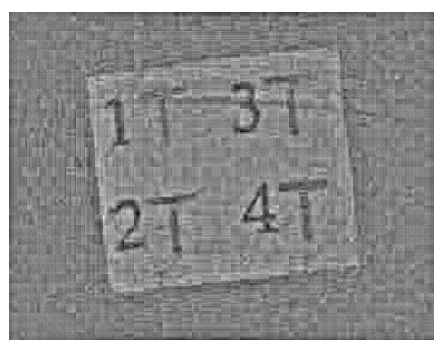

(c) high-frequency part

Figure 1. Underwater image 


\section{IMPROVED MEAN SHIFT FILTERING}

The mean shift algorithm was first proposed by Fukunaga[2] in 1975,but did not get much attention untill 1995, Cheng[3] expanded the application fields by introducing the kernel function, in 2002, Comaniciu[4] applied the algorithm in feature space analysis and got good effect in image filtering and image segmentation. The mean shift vector is defined as:

$$
M_{h}(x) \equiv m_{h}(x)-x, \text { where, } m_{h}(x) \equiv \frac{\sum_{i=1}^{n} K\left(\frac{x_{i}-x}{h}\right) w\left(x_{i}\right) x_{i}}{\sum_{i=1}^{n} K\left(\frac{x_{i}-x}{h}\right) w\left(x_{i}\right)}
$$

,$K(x)$ is the kernel function, $h$ is the bandwith of kernel function, $n$ is the number of samples, $w\left(x_{i}\right) \geq 0$ represents the weights for sample $x_{i}$. Given a point $x$ and tolerance $\varepsilon$, the mean shift algorithm is an iteration process: (1) compute $m_{h}(x)$; (2) if $\left\|m_{h}(x)-x\right\|<\varepsilon$, end process; (3) assign $x$ with $m_{h}(x)$, then go to step (1). When applied for gray image smoothing, each sample represents a 3-D vector $(p, q, I(p, q))$, where $(p, q)$ is the space site of a pixel and $I(p, q)$ is the gray intensity, after mean shift algorithm, $(p, q, I(p, q))$ will converge to $\left(p_{i}, q_{i}, I_{i}\right)$, then assign $I_{i}$ to the pixel whos space site is $(p, q)$, it is obvious that we must perform the process for every pixel to complete the smothing target, and it is computationally expensive.

We make a hypothesis: for mean shift algorithm, the samples, which are very close to each other, will converge to the same center. We can utilize the intermediate result of iteration to expand the number of samples that will converge to the same point, and this will reduce the iteration counts greatly, following is the detail: define original image $I(p, q)$, filtered image $F(p, q)$, binary matrics $H(p, q), H(p, q)=1$ if $F(p, q)$ is assigned, else $H(p, q)=0$;

(1) select a sample $x=(p, q, I(p, q))$,initial a collection $S=\varnothing$, if $H(p, q)=1$, end ; else add $(p, q)$ into $S$;

(2) if there exist a sample $y=\left(p_{m}, q_{m}, I\left(p_{m}, q_{m}\right)\right)$ to make $\operatorname{dis}(x, y)<\varepsilon$ and $H\left(p_{m}, q_{m}\right)=1$, then all the pixels belong to $S$ are assigned value $F\left(p_{m}, q_{m}\right)$, end;

(3) calculate $m_{h}(x)$, add the space site of all samples $x_{n}$ into $S$, where $\operatorname{dis}\left(m_{h}(x), x_{n}\right)<\varepsilon$;

(4) if there exist a sample $z=\left(p_{n}, q_{n}, I\left(p_{n}, q_{n}\right)\right)$ to make $\operatorname{dis}(x, z)<\mathcal{E}$ and $H\left(p_{n}, q_{n}\right)=1$, then all the pixels belong to $S$ are assigned value $F\left(p_{n}, q_{n}\right)$, end;

(5) $\left\|m_{h}(x)-x\right\|<\varepsilon$, surpose $m_{h}(x)=\left(p_{i}, q_{i}, I_{i}\right)$, all the pixels belong to $S$ are assigned value $I_{i}$, end;

(6) assign $x$ with value $m_{h}(x)$, go to $\operatorname{step}(3)$;

In the experiment, we sample the image at a certain interval, and first filter these samples by mean shift, then handle the rest pixels, this method will protect the result from distrotion. Figure 2 shows the comparision of two mean shift algorithm, for a gray image of $256 * 256$, general mean shift carried on 118019 iterations, while the iteration count is only 16409 , about $13.9 \%$, for improved algorithm.

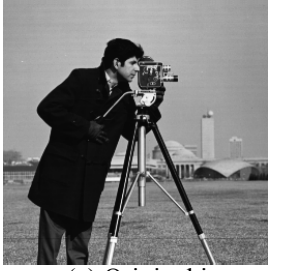

(a) Original image

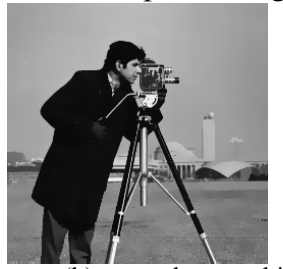

(b) general mean shift

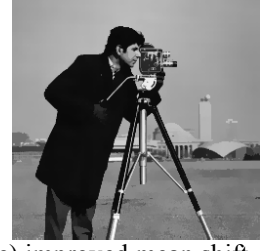

(c) improved mean shift

Figure 2

\section{CONTRAST ENHANCEMENT}

Although noise has been removed, the result is still fuzzy and looks like plagued by fog because of the light absorption and scattering by water molecule. A widely used model for describing the formation of a haze image is: $\mathbf{I}(x)=\mathbf{J}(x) \mathbf{t}(x)+A(1-\mathbf{t}(x))$, where $\mathbf{I}$ is the observed image, $\mathbf{J}$ is the surface radiance, $A$ is the atmospheric light and $\mathbf{t}$ is the transmission rate. According to the model,

$$
\mathbf{J}(x)=A+\frac{(\mathbf{I}(x)-A)}{\mathbf{t}(x)} \text {,if we know } A \text { and } \mathbf{t} \text {,we get } \mathbf{J} \text {. }
$$

HE[5] proposed a dark channel prior to remove haze, according to the prior, the estimated transmission rate is $t(x)=1-\omega \min _{c}\left(\min _{y \in \Omega(x)}\left(\frac{I^{c}(y)}{A^{c}}\right)\right)$, where c stands for the $\mathrm{r}, \mathrm{g}$, b color channel, $\Omega(x)$ is patch centered in $\mathrm{x}, 0<\omega<1$. However, this prior is only suitable for color image and is unable for grayscale image. In this paper, considering for underwater image, We estimate A as:

$$
A=\max \left(I^{\text {dark }}\right) \quad \text {, where } \quad I^{\text {dark }}(x)=\min _{y \in \Omega(x)}(I(y)) \quad \text {.we }
$$

estimate the transmission rate:

$$
t(x)=\left\{\begin{array}{l}
1-\omega \frac{I(x)}{A},(I(x)<=A) \\
1-\omega \frac{A}{I(x)},(I(x)>A)
\end{array}, \omega=0.95\right.
$$

The above equation will darken the zones whos intensity is smaller than $\mathrm{A}$, and will brighten the zones whos intensity is larger than $\mathrm{A}$.

\section{EXPERIMENT RESULTS}

The complete algorithm for underwater image enhancement is as follows: 
(1) decompose the original image into high-frequency part I_H and low-frequency part I_L ;

(2) get I_H_filter by filtering I_H using mean shift;

(3) enhance the contrast of I_H_filter to get I_H_dehaze, enhaze the contrast of I_L to get I_L_dehaze ;

(4) get the final result I_final = I_H_dehaze +I_L_dehaze ;

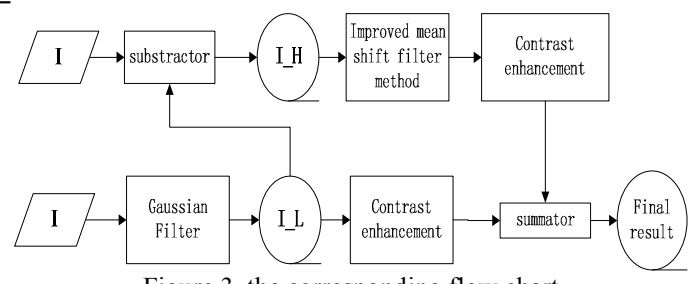

Figure 3. the corresponding flow chart

Figure 4 shows the experiment results for an underwater image which is $320 \times 420$, figure(a) is the original underwater image and the imaging quality is very bad; figure (b) represents the low-frequency part, it reflects the lighting distribution; figure (c) represents the high-frequency part, it reflects the detail and also noise; figure (d) stands for the result of post-filtering by mean shift, noise has almost been removed, but is still misty; figure (e) represents the result of contrast enhancing for figure (d); figure (f) is the final result.

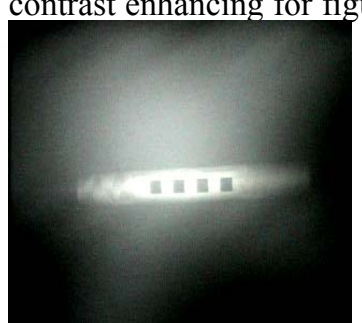

(a) original image

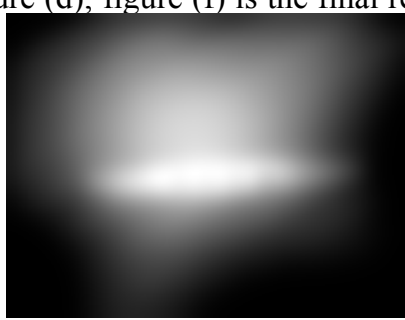

(b)low-frequency part

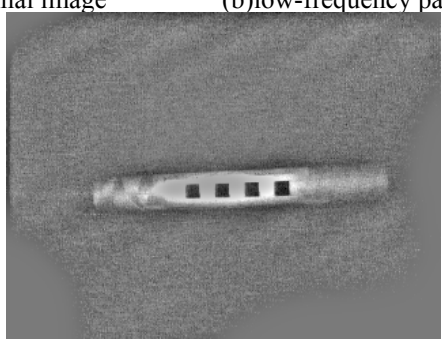

(c) high-frequency part

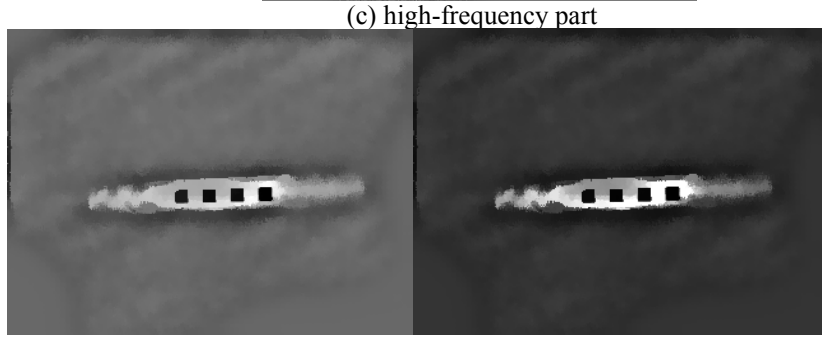

(d) filter by mean shift

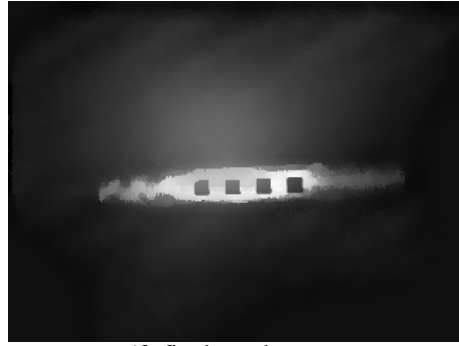

(f) final result

Figure 4. enhancement for underwater image

\section{SUMMARIES}

The quaqlity of underwater image usually is poor due to complex imaging enviroment, and general enhancement technic is helpless. In order to keep the edge and enhance contrast while eliminate noise, we first decompose the original image into high-frequency part and low-frequency part, then filter the high-frequency part by improved mean shift method, and enhance the contrast. For underwater images with structure-object in active illumination environment, experiment results demonstrate the effectiveness of this alogrithm.

\section{ACKNOWLEDGEMENT}

This paper is supported by the National Science Foundation Project (60872076).

\section{REFERENCES}

[1] LAN Guoling, LI Jian, JI Fang. Underwater Image Backscatter Noise Reduction Based on Wavelets. Ocean Technology [J], 2010, vol 29, no 2, PP:43-47.

[2] Fukunaga K, Hostetler L. The Estimation of the Gradient of a Density Function, with Applications in Pattern Recognition. IEEE Trans on Information Theory, vol. 21, no.1, pp. 32-40, Jan 1975.

[3] Yizong Cheng. Mean shift, mode seeking and clustering. IEEE Transactions on Pattern Analysis and Machine Intelligence, vol. 17, no. 8, pp. 790-799, Aug. 2002.

[4] D. Comaniciu, P. Meer, Mean Shift: A Robust Approach Toward Feature Space Analysis. IEEE Transactions on Pattern Analysis and Machine Intelligence, vol. 24, no. 5, pp. 603-619, May 2002.

[5] Kaiming He, Jian Sun, Xiaoou Tang. Single Image Haze Removal Using Dark Channel Prior. IEEE Transactions on Pattern Analysis and Machine Intelligence, 31 Aug. 2010. 